\title{
Experimental and computational study of acceleration response in layered cylindrical structure considering impedance mismatch effect
}

\author{
Sachiko Sueki ${ }^{\mathrm{a}, *}$, Samaan G. Ladkany ${ }^{\mathrm{b}}$ and Brendan J. O'Toole ${ }^{\mathrm{c}}$ \\ ${ }^{a}$ Corresponding Author, Department of Civil and Environmental Engineering, University of Nevada, Las Vegas, \\ 4505 Maryland Parkway, Las Vegas, NV, USA \\ ${ }^{\mathrm{b}}$ Department of Civil and Environmental Engineering, University of Nevada, Las Vegas, NV, USA \\ ${ }^{\mathrm{c}}$ Department of Mechanical Engineering, University of Nevada, Las Vegas, NV, USA
}

Received 30 October 2009

Revised 27 June 2010

\begin{abstract}
Electronic devices, especially those having high performance capabilities, are sensitive to mechanical shocks and vibrations. Failure of such devices in smart projectiles caused by vibrations has been observed. The currently accepted methodology to protect electronic devices in smart projectiles is use of stiffeners and dampers. However these methods are not effective in protecting the electronic devices from high frequency accelerations in excess of 5,000 Hz. Therefore, it is important to find more effective methods to reduce high frequency vibrations for smart projectiles. In this study, layered cylindrical structures are studied experimentally and computationally to understand the effect of impedance mismatch in axial acceleration response under an impact loading. Experiments are conducted by applying impact forces at one end of cylindrical structures and measuring accelerations at the other end. Experimental results suggest that high frequency accelerations in layered structures could be less compared to those in homogeneous cylinders if a returning wave from the end of the projectile does not interfere with the applied impact force. Computational studies using finite element analysis (FEA) verified the experimental results of our interference hypothesis.
\end{abstract}

Keywords: Impact, mitigation, acceleration, impedance, FEA and layered cylindrical structure

\section{Introduction}

Vibrations are, in general, undesired since they may cause damage to mechanical systems and structures. For instance, excessive vibrations can cause undesirable motions, misalignments and disconnection of screws which lead to a faulty performance of a machine or device. Vibrations can also cause variable stresses which lead to damage or failure due to fatigue in structures such as cracking and wearing [1]. Fraser and Gureghian [2] also pointed out that electronic devices, especially those having high performance capabilities, are sensitive to mechanical shocks and vibrations. Similarly, electronic devices in smart projectiles seem to fail because of high frequency vibrations. High accelerations induced during muzzle exit may be a source of electronic component failure in smart projectiles. As mentioned by Frost and Costello [3] and Wilson et al. [4], a key component of a smart projectile must withstand

*Corresponding author. Tel.: +1 702862 5417; Fax: +1 702862 5427; E-mail: sachiko.s@gmail.com. 
high-g loads during launch. In order to increase impact tolerance of electronic devices, the current accepted methods are generally a combination of stiffening and damping elements [5]. However, as Veprik and Babitsky [6] pointed out, these methods cannot protect the electronic devices from high frequencies which typically contain resonant frequencies for sensitive internal components. Therefore, it is important to find more effective methods to reduce high frequency vibrations to protect the electronic devices in smart projectiles.

In order to reduce vibrations in a projectile, design must be simple and robust. Previously, Solaroli et al. [7] studied the attenuation of wave propagation by periodically changing stiffness of a shell structure adding rings around it. They concluded that the wave propagation attenuates over certain frequency bands as a result of impedance changes generated by the periodic discontinuities in stiffness. Similarly, Toso and Baz [8] studied wave propagation characteristics in long cylinders using periodicity, variable geometry, material properties and functionally graded materials (FGM). They concluded that the differences in geometry significantly affect wave propagation over certain wave frequencies. However, if the geometry of a structure is a constraint, FGM can be employed to improve the attenuation of wave propagation. Similar to periodic structures and FGM, other materials such as laminated composites have also been studied by several researchers. Li et al. [9] conducted experiments using FGM and layered materials and concluded that interfaces play a crucial role in dynamic problems. Tasdemirci and Hall [10] studied the effect of the material combinations using ceramic, aluminum and copper which have significant differences in their impedances. Their experimental and computational work demonstrated that impact on multi-layer materials can produce severe stress heterogeneities at the interfaces. Similarly, there have been many other reports in layered composite materials since they have an advantage of having low density with resistance to impacts. However, as reviewed by Bert [11] and Abrate [12] most of the research on the laminated composites are focused in damage resistance and tolerance aspects of usage in lightweight armor to defeat projectiles. There are few publications on acceleration wave propagation in layered composites. Based on a well know phenomenon (when elastic wave passes through solid materials and meets interface of layers, a part of the wave is reflected from the boundary and the rest is transmitted through based on differences in impedance [13-15]), it is clear that layered composites whose interfaces have high impedance mismatch change their acceleration response. Therefore in this study, we focused on layered cylindrical structures and studied experimentally and computationally in order to understand the effect of impedance mismatch on acceleration response under impact loadings.

\section{Two types of cylindrical structures}

Short and long cylindrical structures are used in the study to understand the effects of impedance mismatch in the axial wave propagation. Both structures are built using either one material (without impedance mismatch) or two materials (with impedance mismatch) and acceleration responses are compared between them. The short structure is chosen based on the experimental projectiles used in the past research [16-18]. The projectiles used in their study are cylinders with a hollow section at the center. Those are made of aluminum or aluminum and steel, and have 101.09 $\mathrm{mm}$ diameter and $152 \mathrm{~mm}$ length. Therefore, in this study, aluminum is selected as the baseline material for both short and long structures. The short structures have overall lengths of $152 \mathrm{~mm}$ and diameters of $101.6 \mathrm{~mm}$, and are made of either aluminum or aluminum and polycarbonate. The second structure is longer and has smaller diameter than the first type. The overall lengths and diameters of the long structures are approximately $2.26 \mathrm{~m}$ and $25 \mathrm{~mm}$, respectively. The structures are made of either aluminum or aluminum and nylon. The second material of each type of structure is chosen based on significantly different impedance compared to aluminum, strength, availability, ease of fabrication, and low cost. Note that the material properties of polycarbonate and nylon are very similar (Table 1). Detailed descriptions of all the structures used in the study are given in later sections.

\section{Experimental studies}

The experiments are conducted by applying impacts at one end and measuring accelerations on the other end of the suspended cylindrical structure as shown in Fig. 1. The short structure is suspended by ropes at two ends with the help of metal bands and the long structure is simply suspended by ropes at two ends. Suspension length of 
Table 1

Material properties used in the computational study

\begin{tabular}{llccccc}
\hline Structure & Material & $\begin{array}{c}\text { Density } \\
\rho\left(\mathrm{kg} / \mathrm{m}^{3}\right)\end{array}$ & $\begin{array}{c}\text { Young's modulus } \\
E(\mathrm{GPa})\end{array}$ & $\begin{array}{c}\text { Poisson's ratio } \\
\mu\end{array}$ & $\begin{array}{c}\text { Yield strength } \\
\sigma_{y}(\mathrm{MPa})\end{array}$ & $\begin{array}{c}\text { Impedance } \\
Z\left(\mathrm{~kg} / \mathrm{m}^{2} \mathrm{~s}\right)\end{array}$ \\
\hline \multirow{2}{*}{ Short } & Polycarbonate & 1848.5 & 4.27 & 0.35 & 62.0 & $2.81 \mathrm{E}+06$ \\
& Aluminum & 3168.1 & 68.00 & 0.33 & 250.0 & $1.47 \mathrm{E}+07$ \\
\multirow{2}{*}{ Long } & Aluminum & 2761.7 & 68.00 & 0.33 & 250.0 & $1.37 \mathrm{E}+07$ \\
& Nylon & 1435.0 & 1.90 & 0.35 & 62.4 & $1.65 \mathrm{E}+06$ \\
\hline
\end{tabular}

${ }^{a}$ Modified density to match with the weight of experimental structure.

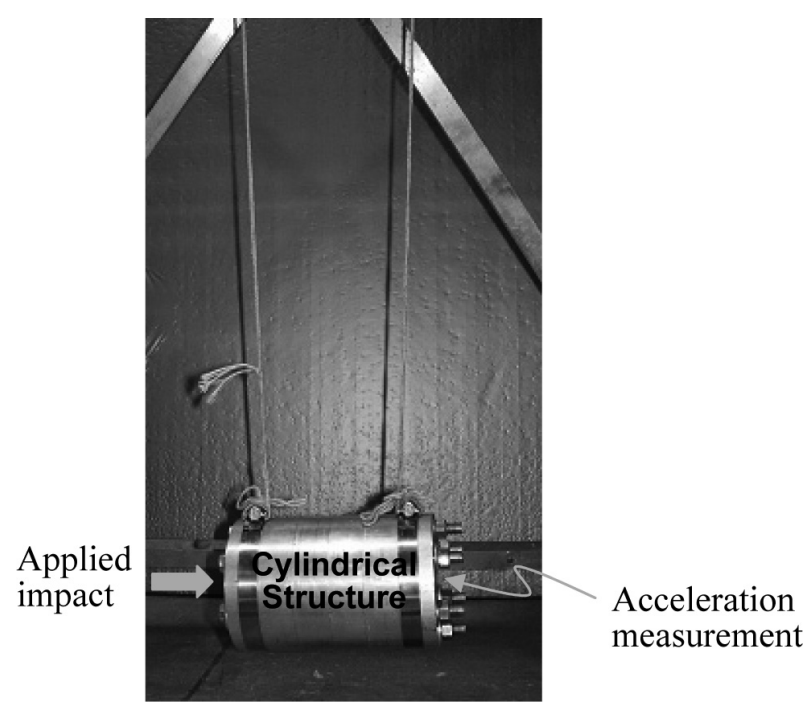

Fig. 1. Experimental setup showing a short cylindrical structure.

approximately $1 \mathrm{~m}$ is used to reduce the resistance to structure's movement from the ropes. An impulse hammer (PCB 086D05) is used to create impacts while accelerations are measured using accelerometers, PCB 352C22 or Dytran $3200 \mathrm{~B}$, depending on the acceleration response. The data is obtained using an oscilloscope (Yokogawa DL750) by connecting the accelerometer and the impulse hammer through a current source power unit (Dytran 4103C). The impulse hammer has $0.23 \mathrm{mV} / \mathrm{N}$ sensitivity and measurement range of $\pm 22 \mathrm{kN}$. The accelerometer, PCB $352 \mathrm{C} 22$ has sensitivity, measurement and frequency ranges of $10 \mathrm{mV} /\left(\mathrm{ms}^{-2}\right), \pm 4,900 \mathrm{~m} / \mathrm{s}^{2}$ and $10 \mathrm{kHz}$, respectively. Similarly, Dytran 3200B has sensitivity, measurement and frequency ranges of $5 \mathrm{E}-3 \mathrm{mV} /\left(\mathrm{ms}^{-2}\right), \pm 980,000 \mathrm{~m} / \mathrm{s}^{2}$ and $20 \mathrm{kHz}$, respectively. Both hard and medium tips of the impulse hammer are used in the experiments. The acceleration responses reported in this paper are representative results obtained from number of test results. A manually applied impulse hammer has a possibility to change its magnitude and time duration every time a test is conducted. Therefore, acceleration responses obtained from similar impact forces are compared and representative acceleration responses have been selected. Note that when the impact forces were reduced, acceleration responses were also reduced accordingly. Also, whenever the impact caused waggling side movements in the structure, the data was discarded.

Acceleration responses are recorded at every $5 \mu$ s and frequency domain responses are calculated by the Fast Fourier Transform (FFT) using the software Altair Hyperview from the recorded time domain response. Final results of time domain response are filtered using a low-pass filter. The frequency used for filtering is noted in the figures since cutoff frequency changes, depending on the structure type.

As described before, two different types of cylindrical structures are used in the experiments. Each structure has different layer configurations. In the short structure, there are three layer configurations (Fig. 2). Each configuration is made of two end plates and four rings in the middle which are assembled by six $9.525 \mathrm{~mm}$ diameter bolts, preloaded with 21 or $34 \mathrm{~N}$-m torque. Both end plates have $101.6 \mathrm{~mm}$ diameter, and each ring has outside diameter of $101.6 \mathrm{~mm}$ and inside diameter of $50.8 \mathrm{~mm}$. All plates are $25.4 \mathrm{~mm}$ thick. Using two end plates and 4 rings made of different 
Table 2

Structural types and configurations

\begin{tabular}{lll}
\hline Type & Structure & Configuration \\
\hline Short-0 & Short Cylinder & Aluminum only \\
Short-1 & & 1 polycarbonate layer between aluminum layers \\
Short-2 & & 2 polycarbonate layers between aluminum layers \\
Long-0 & Long Cylinder & Aluminum only \\
Long-1 & & Nylon between aluminum layers \\
\hline
\end{tabular}

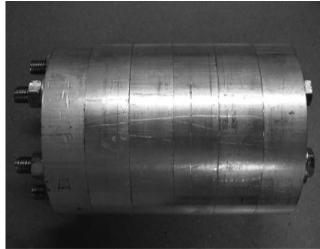

(a) Short -0

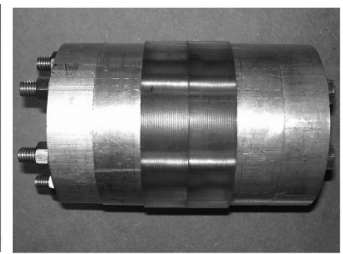

(b) Short-1

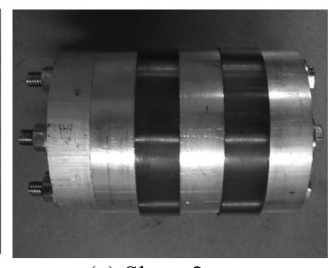

(c) Short-2

Fig. 2. Three different layer configurations of the short structure used in the experiments; (a) aluminum only, (b) aluminum with a polycarbonate layer in the middle and (c) aluminum and two layers of polycarbonate.

(a) Long-0

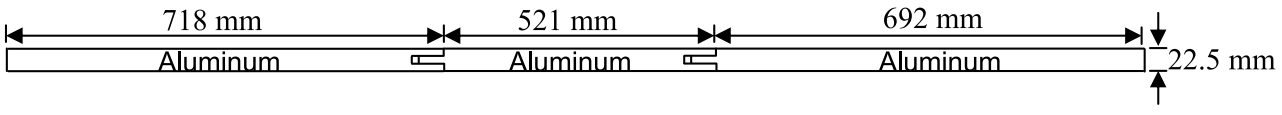

(b) Long-1

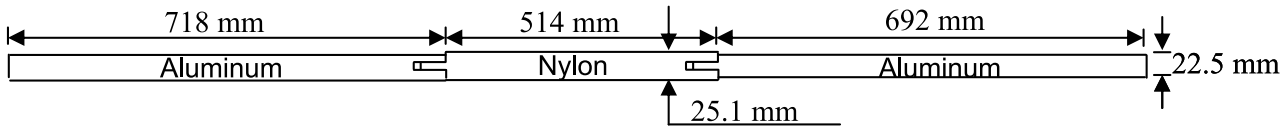

Fig. 3. Two different layer configurations of the long structure and their dimensions used in the experiments; (a) all three parts are made of aluminum, (b) two ends are made of aluminum and the center part is made of nylon.

material, three different layer configurations are built. The first configuration, 'Short-0', is made of all aluminum parts. The second configuration, 'Short-2', has one layer made of two polycarbonate rings (double length) at the middle of the structure. Finally, the last configuration, 'Short-3', has two layers of polycarbonate between aluminum parts.

In the long structure, there are two layer configurations as shown in Fig. 3. The long structure is built using three components fastened by a 5/16-24 thread. In the first case, 'Long-0', all three parts of the cylindrical structure are made of aluminum. In the second case, 'Long-1', the center part of the structure is made of nylon while ends are made of aluminum. Exact dimensions of the structures are shown in Fig. 3 since dimensions of each structure are slightly different because of availabilities of aluminum and nylon long rods and fabrication of threads.

Configurations of each structure are summarized in Table 2. From here on, each configuration is called by the type as listed in Table 2.

\section{Computational studies}

\subsection{Simulation of experimental condition}

Two different types of cylindrical structures which are similar to those used in the experiments are modeled using the software, Altair HyperMesh (Fig. 4). 14,496 and 17,952 eight-node solid hexahedron elements are used for the short and long cylindrical structures, respectively. Accelerations are then computed at the center node of one end by applying impact forces at nine center nodes of the other end using the finite element software, LS-DYNA. 


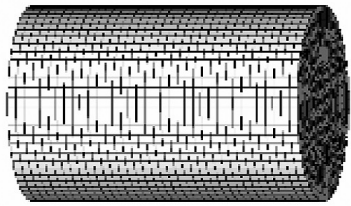

(a) Short-0

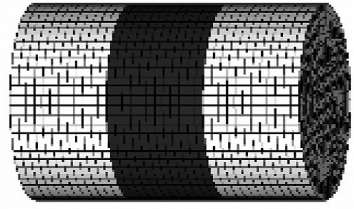

(b) Short-1

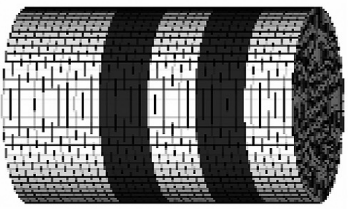

(c) Short-2

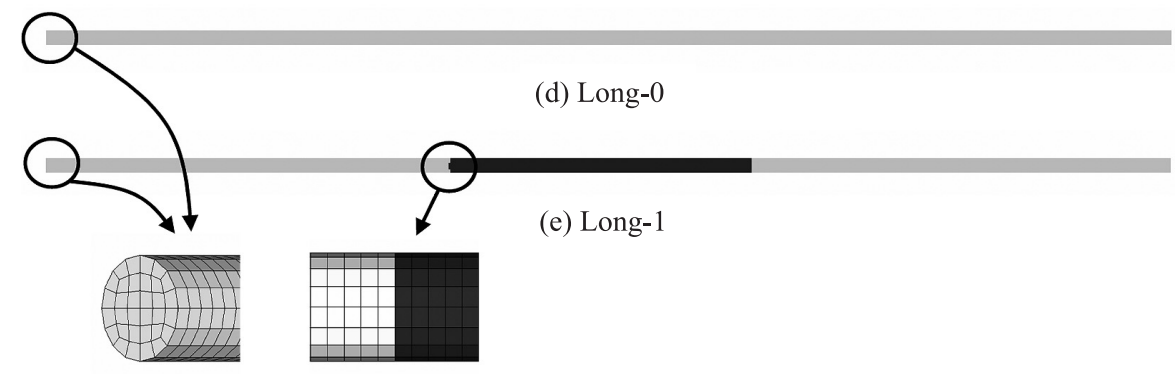

Fig. 4. Simulation models of the short (figures $\mathrm{a}, \mathrm{b}$ and $\mathrm{c}$ ) and long (figures $\mathrm{d}$ and e) cylindrical structures. Different shades represent different materials; (a) a short structure made of aluminum only (b) a short structure made of aluminum and one layer of polycarbonate at the middle (c) a short structure made of aluminum and two layers of polycarbonate (d) a long structure made of aluminum only (e) a long structure made of aluminum and nylon at the middle.

The impact forces used in the models are obtained from the experiments. One-point Gaussian quadrature is used to carry out the volume integration and constant stress solid element type is used in the computational process based on substantial savings in computer time [19,20]. Computational study follows the same configurations as the experiments. The model uses shared nodes between two different materials unlike experimental structures which are detachable. Material properties used in the model are tabulated in Table 1. Note that the density of each material is modified to match with the weight of the experimental structures since model uses simplified structures such as no bolts and no minor differences in dimensions. There are a few literatures reporting effects of bolted connections such as reducing acceleration responses and changing excitation frequencies of structures [21-24]. However, inclusion of bolted joints in a model increases uncertainty in the results since they can be modeled in a number of different ways and there are several options to add for pre-tensions in bolts. Therefore, depending on the selection, models provide different results and degree of complications increase. For that reason, bolted joints are omitted in our models because our focus is to study the effect of material differences in acceleration rather than effect of bolted connections. Also, as shown in Figure 4e, "Long-1" model does not include diameter differences between aluminum and nylon parts unlike in the experimental structure. This was simplified to reduce computational time since there was only a minor change in acceleration response when the larger diameter effects are included. Eigen frequencies of each modeled structures are also obtained using Optistruct, Altair HyperMesh software.

\subsection{Effect of applied impact forces}

Different magnitudes of impact forces with variations in impact durations are used to study effect of applied impact force on acceleration responses of the cylindrical structures. The different impact forces are applied as a half sine curve at the end of the short and long structural models. One primary focuses of this study is the impact duration. By increasing the impact duration, an applied impact may interfere with the wave reflected back from the other end of the structure. The acceleration response affected by the interference between an applied impact and returning wave is studied. As reported by Berman [25], time resolution of loading function affects the response results showing a significant transient at each point in the input function. Therefore, interval of input forces is determined by trial and error to assure time resolution does not affect the response. Impacts applied to the structures have the exact same impulses. This was achieved by altering the magnitude of impact forces based on the impact duration. The models used in this study duplicate the short and long structures used in the experimental simulation including the boundary conditions. Detailed description of applied impulses is given in the results section. 
Table 3

Excitation frequencies $(\mathrm{Hz})$ observed in FFT response of experimentally obtained acceleration results of the short structures

\begin{tabular}{cccccccc}
\hline Applied torque & \multicolumn{3}{c}{ Hard tip impact } & & \multicolumn{3}{c}{ Medium tip impact } \\
\cline { 2 - 4 } \cline { 7 - 8 } & Short-0 & Short-1 & Short-2 & & Short-0 & Short-1 & Short-2 \\
\hline $21 \mathrm{~N}-\mathrm{m}$ & 7,380 & 5,260 & 5,800 & & 7,420 & 5,360 & 5,900 \\
$34 \mathrm{~N}-\mathrm{m}$ & 9,030 & 5,620 & 6,310 & & 9,100 & 5,650 & 6,340 \\
\hline
\end{tabular}

\section{Results}

\subsection{Experimental results}

\subsubsection{The short cylindrical structure}

The experimental results of the short cylindrical structures are shown in Figs 5 to 8 . Three different configurations were used to investigate the acceleration response under two different impact tips. Additionally, two different bolt tensions ( $21 \mathrm{~N}-\mathrm{m}$ and $34 \mathrm{~N}-\mathrm{m}$ torque) were used to study the effect of bolt tensions to acceleration responses. As clearly seen in Fig. 5, applied impact of each structure has identical forces. Figure 6 shows FFT response of applied impacts. Range of excitation frequencies was increased from approximately 3,000 to $8,000 \mathrm{~Hz}$ with a change in impact hammer tips from medium to hard. However, change of the structural configurations did not affect the range of excitation frequencies. The peak magnitudes of applied force using the hard and medium tips were approximately 10,000 and $3,800 \mathrm{~N}$, respectively. Figure 7 shows the FFT responses of all the configurations up to a frequency of $10,000 \mathrm{~Hz}$. There were no observable peaks in the FFT response beyond 10,000 Hz. Therefore, the time domain responses shown in Fig. 8 were filtered at cutoff frequency of 10,000 Hz. Top two figures in Fig. 8 show acceleration responses obtained applying the hard tip impact to the structure fastened with bolt tension 21 and $34 \mathrm{~N}-\mathrm{m}$ torque, respectively. Bottom two figures show acceleration response obtained by applying the medium tip impact fastened with 21 and $34 \mathrm{~N}-\mathrm{m}$ torque, respectively. All four figures show that 'Short-0' had the lowest accelerations followed by 'Short-1'. The highest acceleration was observed in 'Short-2'. The applied torque to fasten the bolts did not show much effect on the magnitude of acceleration response. However, the peak in FFT response shifted to higher frequency for all the layer cases when torque was increased from $21 \mathrm{~N}-\mathrm{m}$ to $34 \mathrm{~N}-\mathrm{m}$ (Fig. 7). The peak values were observed approximately at 7,400 Hz, 5,300 Hz and 5,800 Hz in 'Short-0', 'Short-1' and 'Short-2' with $21 \mathrm{~N}-\mathrm{m}$ torque, respectively. Similarly, 'Short-0', 'Short-1', and 'Short-2' with $34 \mathrm{~N}-\mathrm{m}$ torque showed peaks at 9,000 Hz, 5,600 and 6,300 Hz. Peak frequencies are tabulated in Table 3. Slightly higher excitation frequencies are observed in the medium tip impact cases compared to the hard tip cases but the differences are almost negligible. Even if a different torque was used to fasten the structure, the lowest excitation frequency was always observed in 'Short-1' followed by 'Short-2' and 'Short-0'. Magnitude of the peaks in the FFT response did not show a consistent trend. For the hard tip impact, the largest magnitude of the peak was observed in 'Short-2' followed by 'Short-1' and 'Short-0' for both 21 and $34 \mathrm{~N}-\mathrm{m}$ torque cases. However, for the medium tip impact, the largest magnitude of the peak was observed in 'Short-2' for $21 \mathrm{~N}-\mathrm{m}$ torque case and 'Short-1' for $34 \mathrm{~N}-\mathrm{m}$ torque case. For both torque cases, the magnitude of the peak for 'Short-0' was smaller than the one for 'Short-2'.

\subsubsection{The long cylindrical structure}

Figures 9 and 10 show the experimental results obtained from the long cylindrical structures. Similar to the short structural cases, two different forces, hard and medium tip impacts, were applied. The hard and medium tip impacts had magnitudes of approximately 5,300 and 2,100 N, respectively (Fig. 9). Similar to the short cylindrical structural case, FFT response of impact forces showed increases in range of excitation frequencies with a change in the impact hammer tip from medium to hard. However, any change in structural configurations did not alter the range. Ranges are approximately $3,000 \mathrm{~Hz}$ for the medium and $7,000 \mathrm{~Hz}$ for the hard tip. The acceleration of the hard tip impact shows that the long cylinder made of all aluminum (Long-0), had slightly higher acceleration than the cylinder made of aluminum and nylon (Long-1; Fig. 9). This difference was more prominent for the medium tip impact cases (Fig. 9). The acceleration results were filtered at $5,000 \mathrm{~Hz}$ based on the peaks observed in FFT responses. Unlike the FFT response observed in the short structure, the highest peak in FFT response obtained from the hard and medium tip impacts appeared at different frequencies (Table 4). Also, there are several clearly observable peaks in 

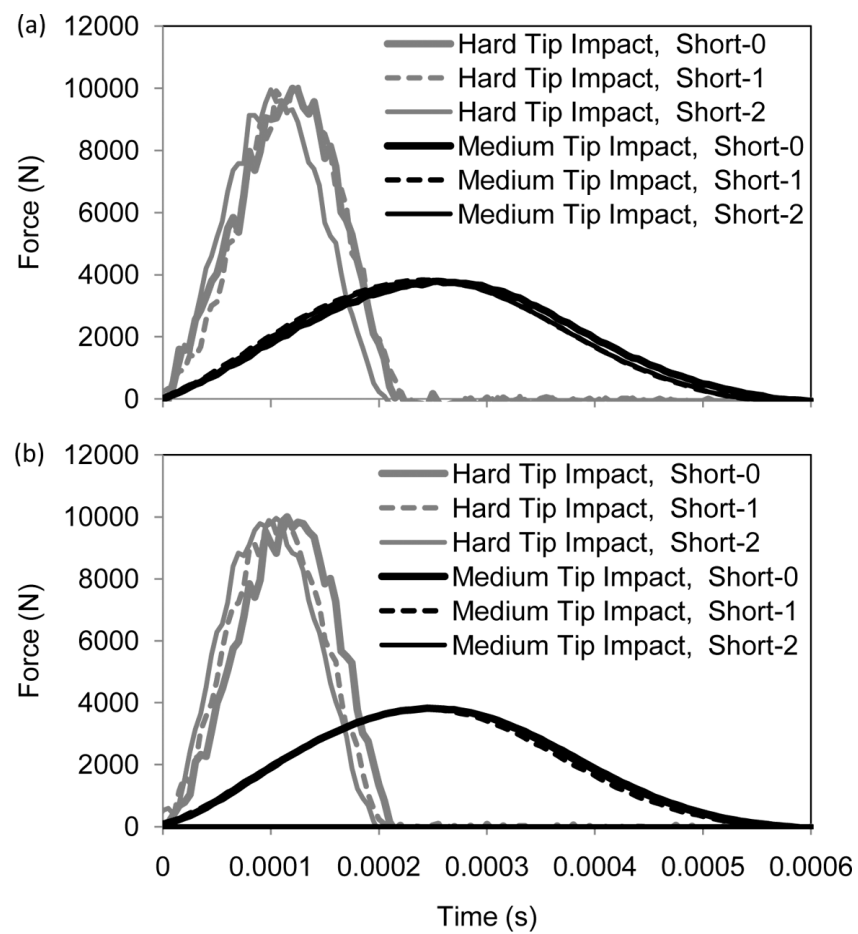

Fig. 5. Applied impact forces on experimental short structures fastened with bolt torque of (a) $21 \mathrm{~N}-\mathrm{m}$ and (b) $34 \mathrm{~N}-\mathrm{m}$.
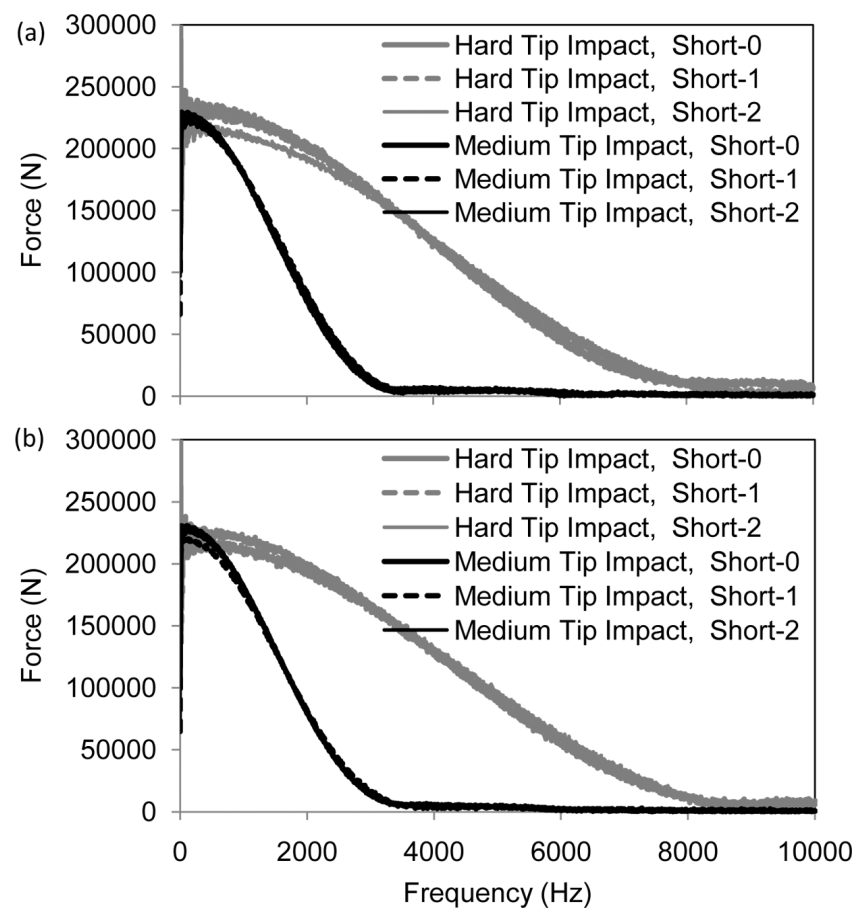

Fig. 6. FFT response of experimentally applied impact force on the short structure fastened with bolt torque of (a) $21 \mathrm{~N}-\mathrm{m}$ and (b) $34 \mathrm{~N}$-m. 


\section{Table 4}

Excitation frequencies $(\mathrm{Hz})$ observed in FFT response of experimentally obtained acceleration results of the long structures. Frequencies are given in descending order of their magnitude

\begin{tabular}{ccccc}
\hline \multicolumn{2}{c}{ Hard tip impact } & & \multicolumn{2}{c}{ Medium tip impact } \\
\cline { 1 - 2 } \cline { 4 - 5 } Long-0 & Long-1 & & Long-0 & Long-1 \\
\hline 3,900 & 3,550 & & 2,520 & 420 \\
2,520 & 420 & & 1,200 & 3,550 \\
1,200 & & & 4,830 & \\
4,830 & & & 3,610 & \\
& & & 3,900 & \\
\hline
\end{tabular}
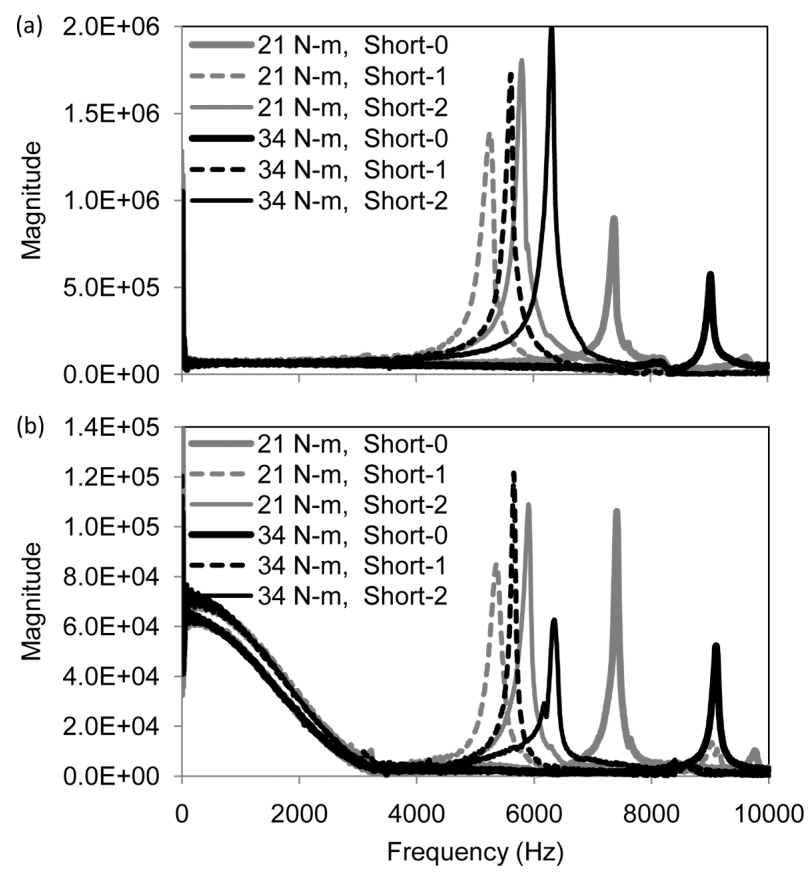

Fig. 7. Experimental results of FFT response of the short structure under impact loading using (a) hard and (b) medium tip impacts.

FFT responses of the long structures while the short structures showed only one clear peak in each configuration. The 'Long-0' configuration had the highest peak at 3,900 Hz for hard and 2,520 Hz for medium tips, while 'Long-1' configuration had highest peaks at $3,550 \mathrm{~Hz}$ for hard and $420 \mathrm{~Hz}$ for medium tips. For both impact cases, 'Long-1' showed the highest peak at lower frequency than that of 'Long-0'.

\subsection{Computational results}

\subsubsection{The short cylindrical structure}

Computational results of the short structure using the impact force obtained from the experiments are shown in Fig. 11 and Table 5. Since structures were modeled with shared nodes between two different materials, effect of the bolt torque forces cannot be included in the model. Therefore, the force curve obtained experimentally using the structure fastened by $21 \mathrm{~N}-\mathrm{m}$ torque are used to compute acceleration responses. Computational results (Table 5) of FFT response of 'Short-0' showed peaks at much higher frequencies (13,120 and 19,020 Hz) compared to the peaks from the experimental results $(\approx 7,400 \mathrm{~Hz}$ for the $21 \mathrm{~N}-\mathrm{m}$ torque case and $9,000 \mathrm{~Hz}$ for the $34 \mathrm{~N}-\mathrm{m}$ torque cases). Therefore, acceleration responses were filtered at cutoff frequency of $20,000 \mathrm{~Hz}$ which are higher than one used for experimental results to include higher excitation frequencies observed in the models. On the 

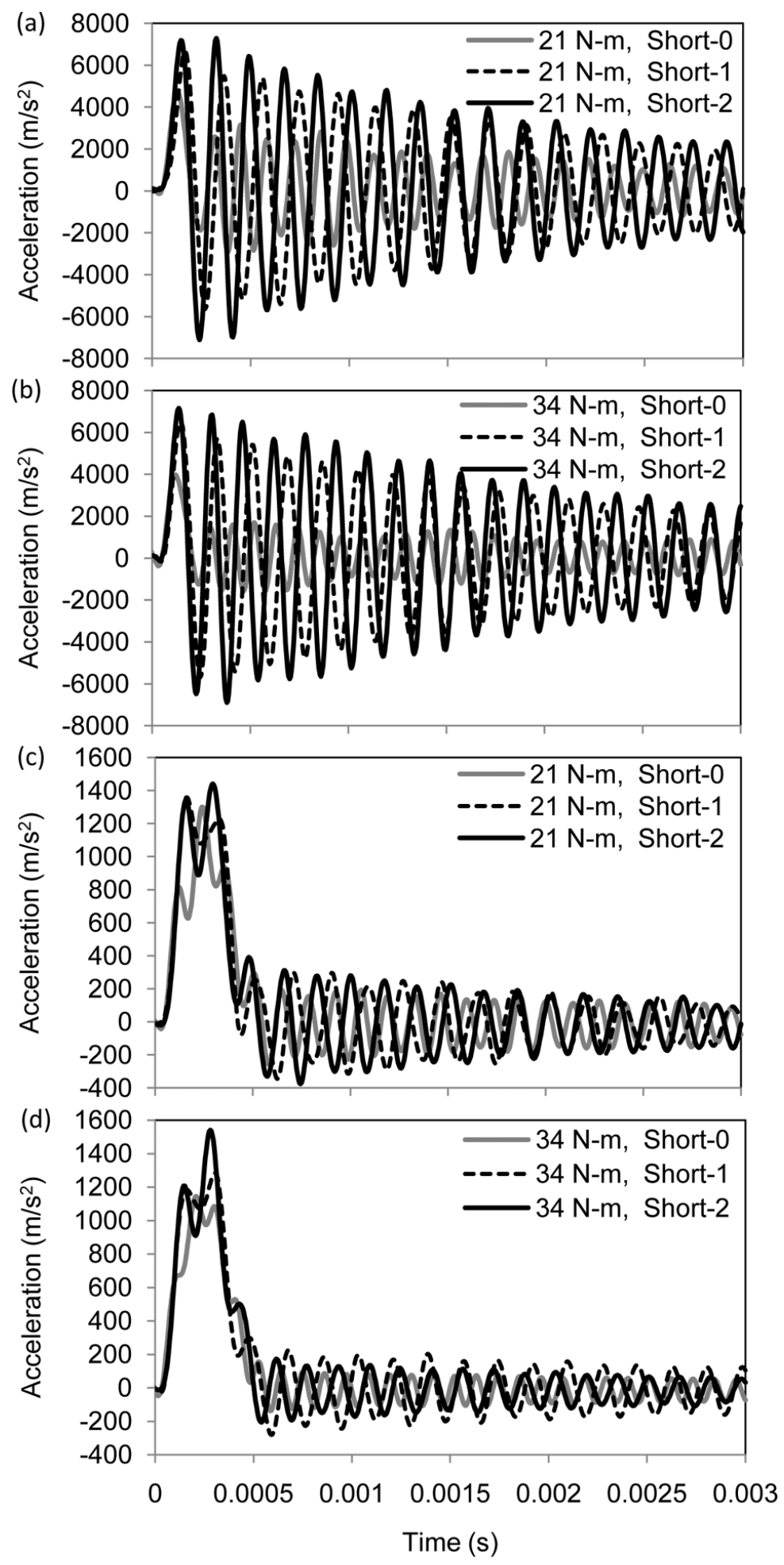

Fig. 8. Experimental results of acceleration response of short structures. (a) the hard tip with bolt tension of $21 \mathrm{~N}$-m, (b) the hard tip with bolt tension of $34 \mathrm{~N}-\mathrm{m}$, (c) the medium tip with bolt tension of $21 \mathrm{~N}-\mathrm{m}$ and (d) the medium tip with bolt tension of $34 \mathrm{~N}-\mathrm{m}$. $10,000 \mathrm{~Hz}$ cutoff frequency is used for low-pass filter. 


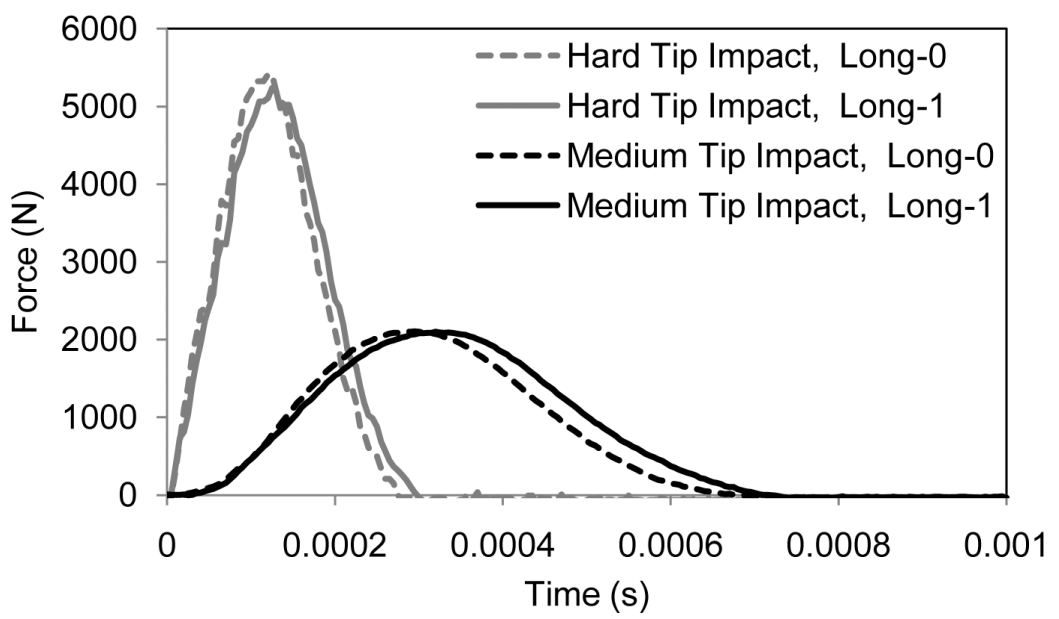

Fig. 9. Applied impact forces on experimental long structures.
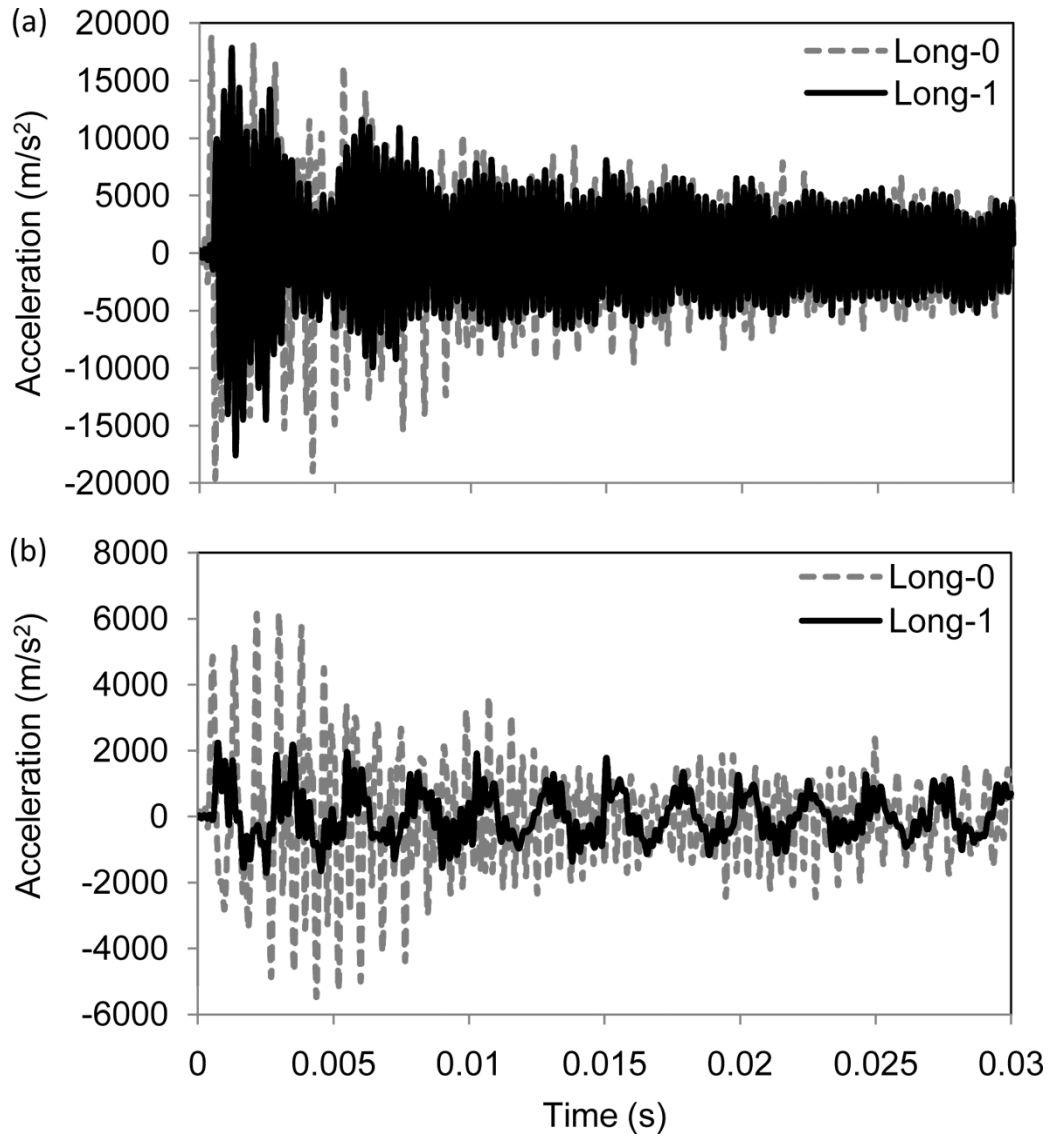

Fig. 10. Experimental results of acceleration responses of long structures under impact loading using (a) hard and (b) medium tip impacts. $5,000 \mathrm{~Hz}$ cutoff frequency is used for low-pass filter. 
Table 5

Excitation frequencies $(\mathrm{Hz})$ observed in FFT response of acceleration results obtained from the experimental simulation of the short structures. Frequencies are given in descending order of their magnitude

\begin{tabular}{ccc}
\hline & \multicolumn{2}{c}{ Hard and medium tip impact } \\
Short-0 & Short- 1 & Short-2 \\
\hline 13,120 & 4,780 & 5,590 \\
19,020 & & \\
\hline
\end{tabular}

Table 6

Eigen frequencies $(\mathrm{Hz})$ of axial mode up to $20,000 \mathrm{~Hz}$ obtained from the short structural models

\begin{tabular}{lrr}
\hline Short-0 & Short-1 & Short-2 \\
\hline 12,600 & 4,600 & 5,360 \\
18,400 & 13,000 & 10,200 \\
& 17,000 & 13,200 \\
& & 14,300 \\
& & 18,600 \\
& & 19,600 \\
\hline
\end{tabular}

Table 7

Excitation frequencies $(\mathrm{Hz})$ observed in FFT response of acceleration results obtained from the experimental simulation of the long structures. Frequencies are given in descending order of their magnitude

\begin{tabular}{|c|c|c|c|}
\hline \multicolumn{2}{|c|}{ Hard tip impact } & \multicolumn{2}{|c|}{ Medium tip impact } \\
\hline Long-0 & Long-1 & Long-0 & Long-1 \\
\hline 2,620 & 3,540 & 1,310 & 300 \\
\hline 3,930 & 3,170 & 2,620 & 1,180 \\
\hline 1,310 & 3,800 & 3,930 & 3,540 \\
\hline 5,240 & & & \\
\hline 6,550 & & & \\
\hline
\end{tabular}

Table 8

Eigen frequencies $(\mathrm{Hz})$ of axial mode up to $10,000 \mathrm{~Hz}$ obtained from the long structural models

\begin{tabular}{cc}
\hline Long-0 & Long-1 \\
\hline 1,290 & 174 \\
2,570 & 296 \\
3,860 & 420 \\
5,150 & 1,160 \\
6,430 & 2,190 \\
7,720 & 3,110 \\
9,000 & 3,480 \\
& 3,740 \\
& 4,510 \\
& 5,520 \\
& 6,480 \\
& 6,930 \\
& 7,210 \\
& 7,830 \\
& 9,130 \\
\hline
\end{tabular}

other hand, computational results of 'Short-1' and 'Short-2' had slightly lower excitation frequencies (4,780 and $5,590 \mathrm{~Hz}$, respectively) compared to the experimental results. Acceleration in 'Short- 0 ' had the lowest acceleration for both hard and medium tip impacts (Fig. 11). However, the medium tip impact shows that the highest maximum acceleration was observed in 'Short-1', which was not the case for computational results of the hard tip impact and experimental results. Note that the differences in the maximum acceleration obtained from three different layer configurations using medium tip impacts were barely noticeable for both experimental and computational results.

Eigen frequencies of axial mode were also obtained using the same short structural models and results are tabulated in Table 6. Since impacts were applied in axial direction of the cylindrical structures, excitation frequencies observed in the FFT responses (Table 5) were close to the Eigen frequencies of axial mode (Table 6). Also, Eigen frequencies of the first axial mode appeared to be the most excited frequency observed in FFT responses for all the short cases. Eigen frequencies of flexural and twisting modes are also obtained but these frequencies are off from the excitation frequencies observed in FFT responses (results are not shown). 

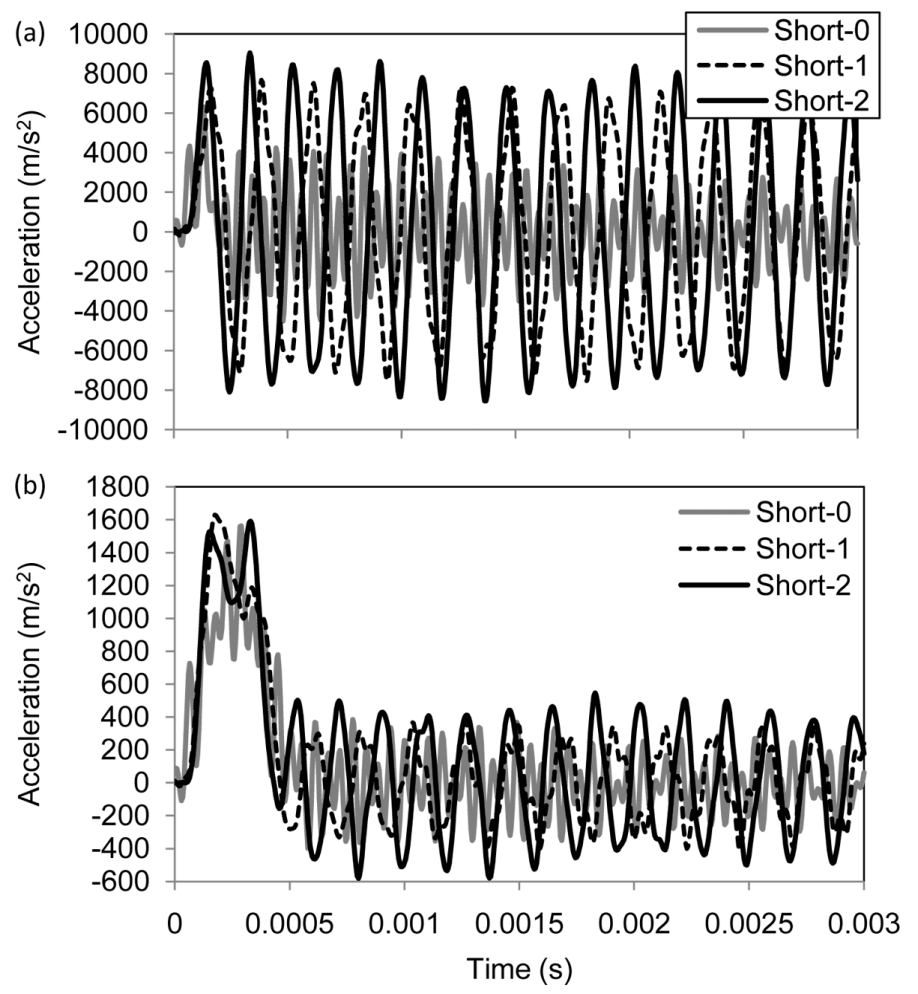

Fig. 11. Computational results of acceleration responses of short structures under impact loading using (a) hard and (b) medium tip impacts. $20,000 \mathrm{~Hz}$ cutoff frequency is used for low-pass filter.

\subsubsection{The long cylindrical structure}

Computational results of the long structure are shown in Fig. 12 and Table 7. As shown in Fig. 12, both the hard and medium tip impacts showed significantly less accelerations in 'Long-1' case compared to 'Long-0'. Similar to experimental result, highest peaks in the FFT response (Table 7) shifted to the lower frequencies when impact tip was changed from hard to medium. However, excitation peaks were not exactly the same as experimental results even though differences were not as significant as those observed in short structures. The 'Long-0' case showed highest peaks at 2,620 and $1,310 \mathrm{~Hz}$ for the hard and medium tips, while 'Long-1' showed highest peaks at 3,540 and $300 \mathrm{~Hz}$ for hard and medium tips, respectively. When the hard tip impact was applied, 'Long-1' had higher excitation frequency $(3,540 \mathrm{~Hz})$ compared to 'Long-0' structure $(2,620 \mathrm{~Hz})$. However, the magnitude of the second excitation frequency $(3,930 \mathrm{~Hz})$ of 'Long-0' was still higher than that of the first excitation frequency $(3,540 \mathrm{~Hz})$ of 'Long-1'. Other than the highest peaks, a number of intermediate peaks are noticeable in FFT results up to nearly $10,000 \mathrm{~Hz}$ for 'Long-0' with the hard tip impact. Therefore, the acceleration responses obtained from computational results of the long structure were filtered with the cut-off frequency of $10,000 \mathrm{~Hz}$ for all the cases.

Table 8 shows Eigen frequencies of axial mode obtained using the same long cylindrical structural models. Unlike short structural cases, the highest magnitude of the excitation frequency observed in all long structures was not close to the Eigen frequency of the first axial mode except in the case of 'Long-0' with medium tip impact.

\subsubsection{Effect of applied impact forces}

\subsubsection{The short cylindrical structure}

Acceleration responses were obtained applying three different impact forces to short and long structures (Fig. 13) in order to study effect of applied impact on acceleration responses. For the short structure, 'Force 1' has the maximum force of $10,000 \mathrm{~N}$ with applied duration of 2.0E-4 seconds similar to the experimental impact applied using the hard tip. The 'Force 2' has the applied impact duration long enough to interfere with the reflected back wave from the end of 'Short-0' structure. The 'Force 3' has the shortest impact duration and the applied impact 
Table 9

Excitation frequencies $(\mathrm{Hz})$ observed in FFT response of acceleration results obtained from the short structural models with three different applied impact forces. Frequencies are given in descending order of their magnitude

\begin{tabular}{|c|c|c|c|c|c|c|c|c|}
\hline \multicolumn{3}{|c|}{ Force 1} & \multicolumn{3}{|c|}{ Force 2} & \multicolumn{3}{|c|}{ Force 3} \\
\hline Short-0 & Short-1 & Short-2 & Short-0 & Short-1 & Short-2 & Short-0 & Short-1 & Short-2 \\
\hline 19,020 & 4,780 & $\begin{array}{c}5,590 \\
10,470\end{array}$ & $\begin{array}{l}13,120 \\
19,020\end{array}$ & $\begin{array}{c}4,780 \\
17,900\end{array}$ & $\begin{array}{c}5,590 \\
10,470\end{array}$ & $\begin{array}{l}13,120 \\
19,020\end{array}$ & $\begin{array}{c}17,900 \\
4,780\end{array}$ & $\begin{array}{c}5,590 \\
10,470\end{array}$ \\
\hline
\end{tabular}
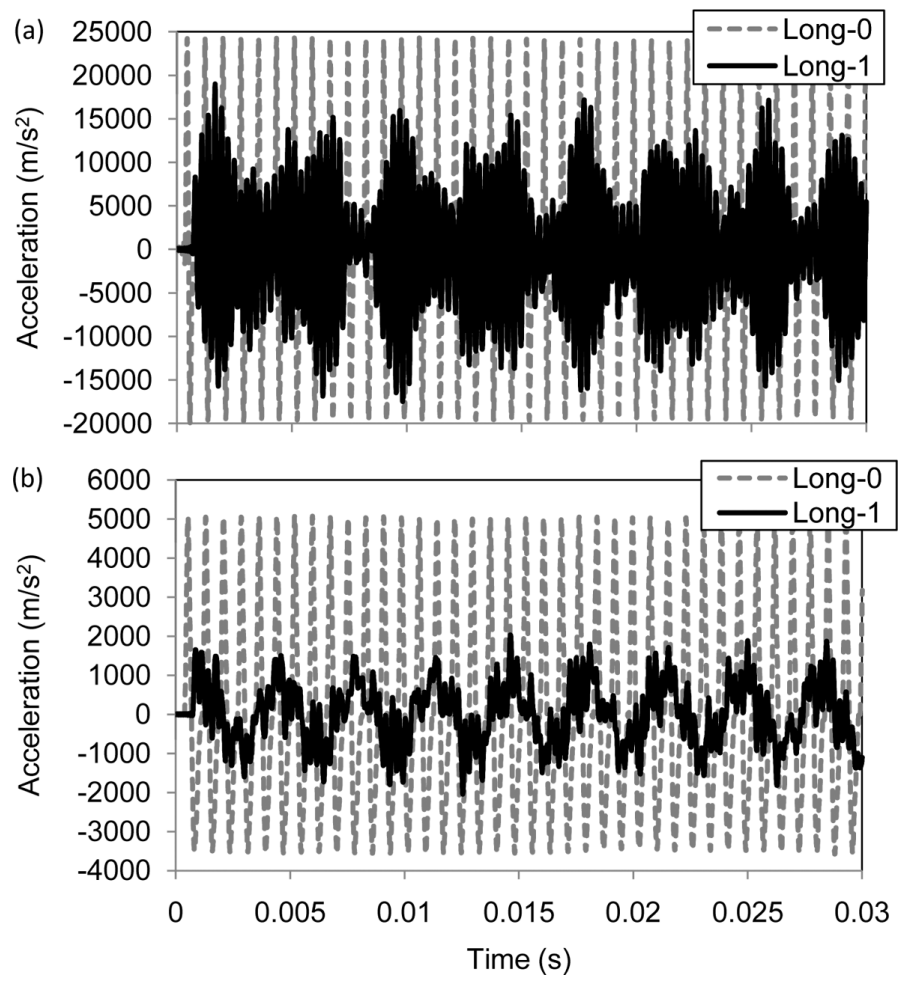

Fig. 12. Computational results of acceleration responses of long structures under impact loading using (a) hard and (b) medium tip impacts. $10,000 \mathrm{~Hz}$ cutoff frequency is used for low-pass filter.

does not interfere with the reflected back wave from the end of any structure ('Short-0', 'Short-1' and 'Short-2'). Maximum magnitude of 19,996 $\mathrm{N}$ for 'Force 2' and 39,992 $\mathrm{N}$ for 'Force 3' were calculated based on the impulse of 'Force 1'. All three forces have similar impulses but range of excitation frequencies increased when magnitudes increased similar to the experimentally applied impact forces. Forces 1, 2 and 3 have the ranges of approximately $6,000,16,000$ and $32,000 \mathrm{~Hz}$, respectively. Interference between the applied force and the reflected back wave was determined based on material wave speed and length of structures. Wave speeds of aluminum, polycarbonate and nylon are approximately $5,000 \mathrm{~m} / \mathrm{s}, 1,500 \mathrm{~m} / \mathrm{s}$ and 1,200 m/s, respectively. Single lap traveling times of 'Short-0', 'Short-1' and 'Short-2' are about 6.1E-5, 1.1E-4 and 1.1E-4 seconds, respectively. Therefore, the impact with duration of 2.0E-4 seconds (Force 1) interferes with reflected wave in all three configurations while impact duration of 5.0E-5 seconds (Force 3 ) does not interfere in any of the cases.

As shown in Fig. 14a, the acceleration results obtained from 'Force 1' duplicate closely to the experimental result. The lowest maximum acceleration was observed in 'Short-0' followed by 'Short-2' and 'Short-1'. However, acceleration results of 'Force 2' showed that 'Short-0' had the highest maximum acceleration followed by 'Short-1' although differences in maximum accelerations of all three cases were small. Fig. 14c shows acceleration response obtained by applying 'Force 3'. The order of maximum accelerations was same as the results obtained from 'Force 

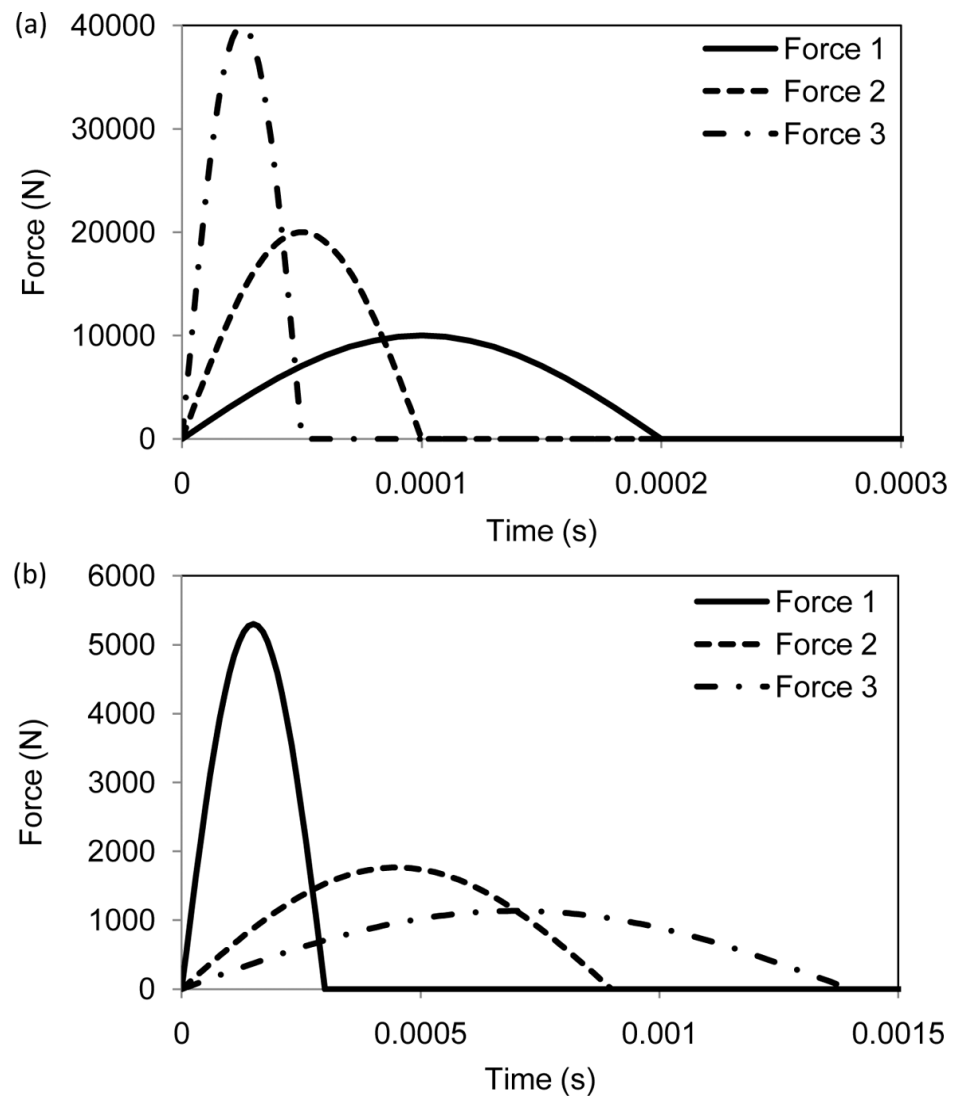

Fig. 13. Applied impact used for (a) short and (b) long structures to study effect of impact forces.

2'. However, 'Force 3' showed significant differences between 'Short-0' and 'Short-2' while 'Short-2' and 'Short-1' showed almost the same maximum acceleration.

Figure 15 shows FFT response of acceleration responses in the short structures using three different applied forces. Results from all forces showed peaks at the exact same frequencies; however the magnitude of each peak was different. The highest peak was in 'Short-1' closely followed by 'Short-2' and significantly lower peak in 'Short-0' when 'Force 1' was applied to the structures (Fig. 15a). In the case of 'Force 2', the highest peak was observed in 'Short-0' followed by 'Short-2' and 'Short-1'. However, all the peaks were close to each other. As shown in Figure 15c, 'Force 3' showed distinctly higher peak in 'Short-0' followed by 'Short-2' and 'Short-1'. Similar to 'Force 1' and 'Force 2', the difference in the magnitude of the peaks between 'Short-2' and 'Short-1' was small. Excitation frequencies in the FFT responses are tabulated in Table 9. When the highest peaks among all the excitation frequencies in each structure were compared, 'Short-0' had the highest frequency among three structural configurations when either the 'Force 1' or 'Force 2' was applied. However, in the case of 'Force 3', 'Short-1' showed the highest excitation frequency even though the magnitude of this excitation frequency was nearly half of the lower magnitude of excitation frequency $(19,020 \mathrm{~Hz})$ observed in 'Short-0'.

\subsubsection{The long cylindrical structure}

The long cylindrical structure model is also used to study the effect of applied impacts. Three applied forces, 'Force 1', 'Force 2' and 'Force 3' shown in Fig. 13b are used to obtain the acceleration responses. 'Force 1' has the maximum force of 5,300 $\mathrm{N}$ with applied duration of 3.0E-4 seconds similar to the experimental impact applied using the hard tip. The 'Force 2' has the applied impact duration long enough to interfere with the reflected back wave from the end of 'Long-0'. Unlike the force applied to the short structure, 'Force 3' has the longest impact duration and the applied impact interferes with the reflected back wave from the ends of both 'Long- 0 ' and 'Long- 1 '. 

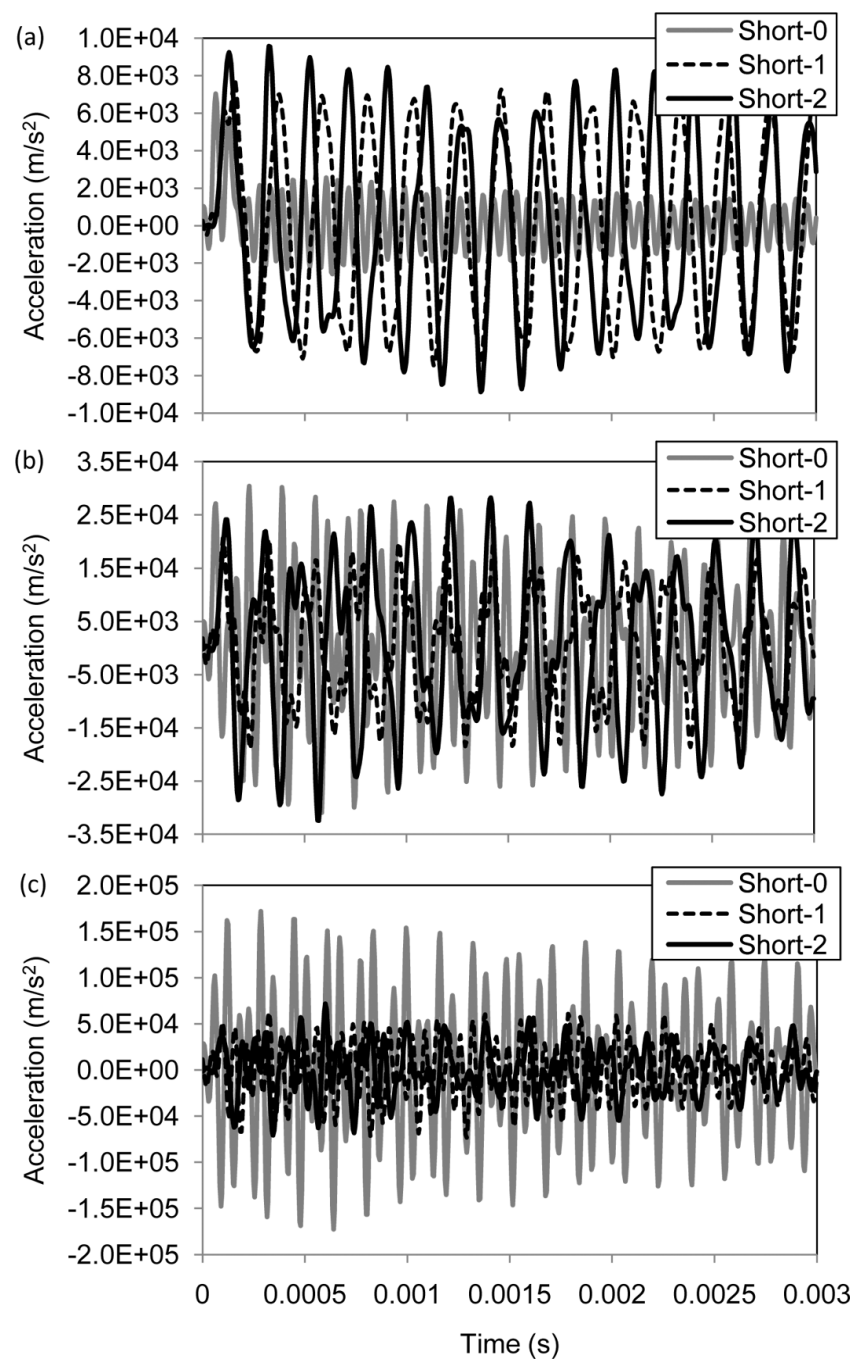

Fig. 14. Computational results of acceleration responses obtained by applying three different impact forces to short structures. Accelerations obtained using (a) Force 1; magnitude of $10,000 \mathrm{~N}$ with 0.0002 seconds impact duration, (b) Force 2; magnitude of $19,996 \mathrm{~N}$ with 0.0001 seconds impact duration and (c) Force 3; magnitude of 39,992 $\mathrm{N}$ with 0.00005 seconds impact duration. 20,000 Hz cutoff frequency is used for low-pass filter.

Single lap traveling time of 'Long-0' is 7.7E-4 seconds while that of 'Long-1' is $1.4 \mathrm{E}-3$ seconds. Therefore, the 'Force 3' impact, with impact duration 1.4E-3 seconds, interferes with reflected wave for both cases. Magnitudes of 'Force 2' and 'Force 3' are 1,766 N and 1,135 N, respectively, which are calculated to have same impulse as 'Force 1'. FFT responses of the applied impacts showed that the range of excitation frequencies for Forces 1,2 and 3 are approximately $6,200,1,600$ and $1,200 \mathrm{~Hz}$, respectively.

In the case of 'Force 1', acceleration was lower in 'Long-1' than that of 'Long-0' (Fig. 16a), which is the same as the experimental result. When the duration of impact force was increased such that the applied impact interferes with the reflected back wave from the other end of 'Long- 0 ' structure but not 'Long- 1 ', the acceleration of 'Long- 0 ' was barely higher than that of 'Long-1' (Fig. 16b). When the impact duration was further increased, 'Long-1' showed higher acceleration response than that of 'Long-0' (Fig. 16c).

The highest peak in the FFT responses of 'Force 1' appeared at different frequencies compared to other two forces as shown in Table 10. The result of 'Force 1' had highest peaks at 2,620 Hz and 3,540 Hz for 'Long-0' and 'Long-1', respectively, while 'Force 2' and 'Force 3' showed highest peaks at 1,310 Hz (Long-0) and $300 \mathrm{~Hz}$ (Long-1). Differences in excitation frequencies were also observed when the impact tip was changed from hard 

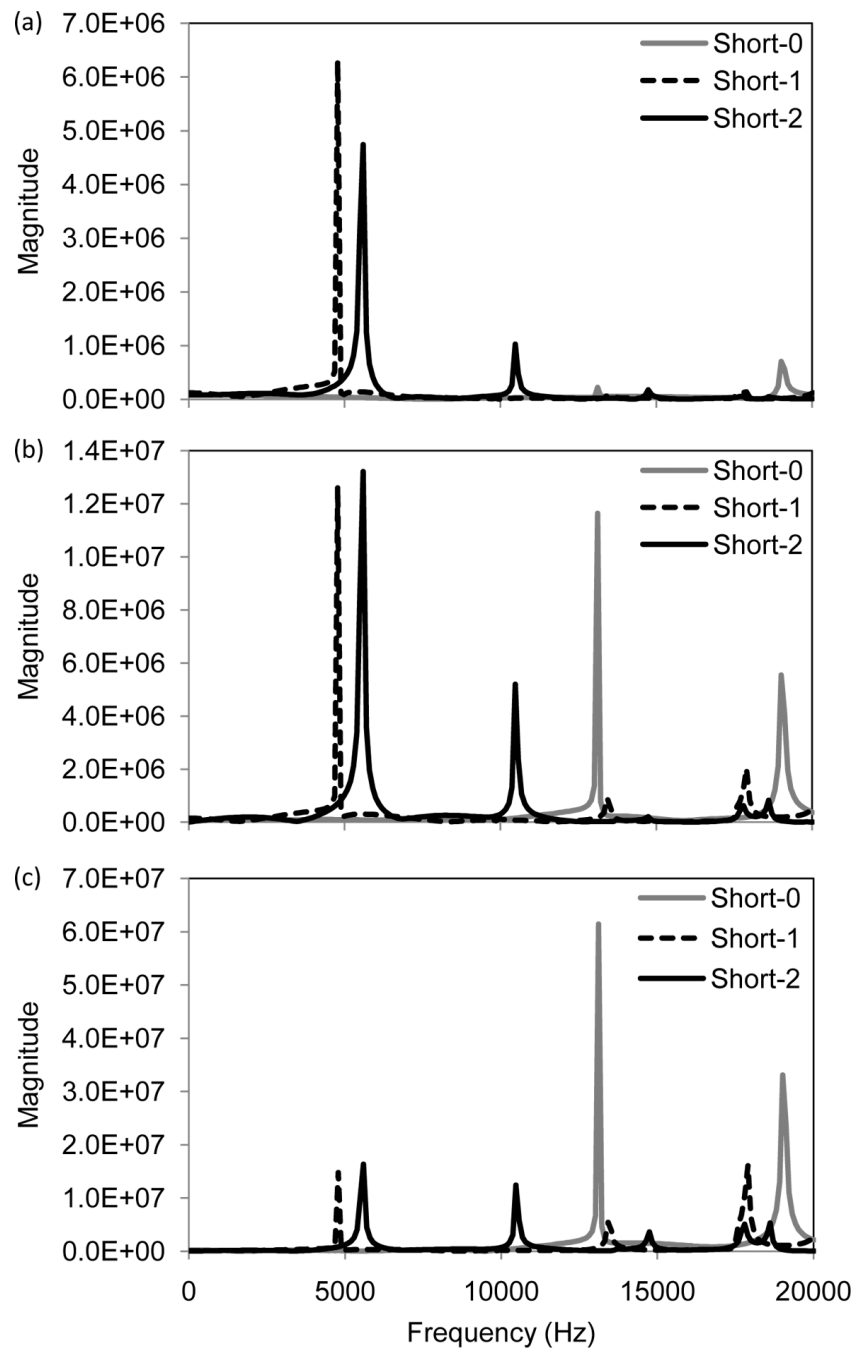

Fig. 15. Computational results of FFT responses obtained by applying three different impact forces to short structures. FFT responses obtained using (a) Force 1; magnitude of 10,000 N with 0.0002 seconds impact duration, (b) Force 2; magnitude of 19,996 N with 0.0001 seconds impact duration and (c) Force 3; magnitude of 39,992 N with 0.00005 seconds impact duration.

to medium tip in the experimental study. The magnitude of the highest peak was higher in 'Long-0' compared to 'Long-1' for 'Force 1' and 'Force 2' cases while 'Force 3' was opposite.

\section{Discussion}

In the experimental investigation of the acceleration response, two different types of cylinder, short and long structures, are used. In the short structure, there are three different layer configurations, 'Short-0', 'Short-1' and 'Short-2'. The order of maximum acceleration response of three different configurations at the end of the structures should be 'Short-0' followed by 'Short-1' and 'Short-2' based on a well known phenomenon that propagating wave gets divided at interface depending on differences in impedance. Since 'Short-0' does not have any interface, entire acceleration wave passes through the structure while 'Short-1' reduces the acceleration wave once and 'Short-2' twice at their interfaces. However, the experimental results showed otherwise. 'Short-0' had the lowest acceleration followed by 'Short-1' and 'Short-2'. This was true for all the torque and impact tip cases in the short structure. On the other hand, the long structure showed results as expected. When there is an interface within the structure 

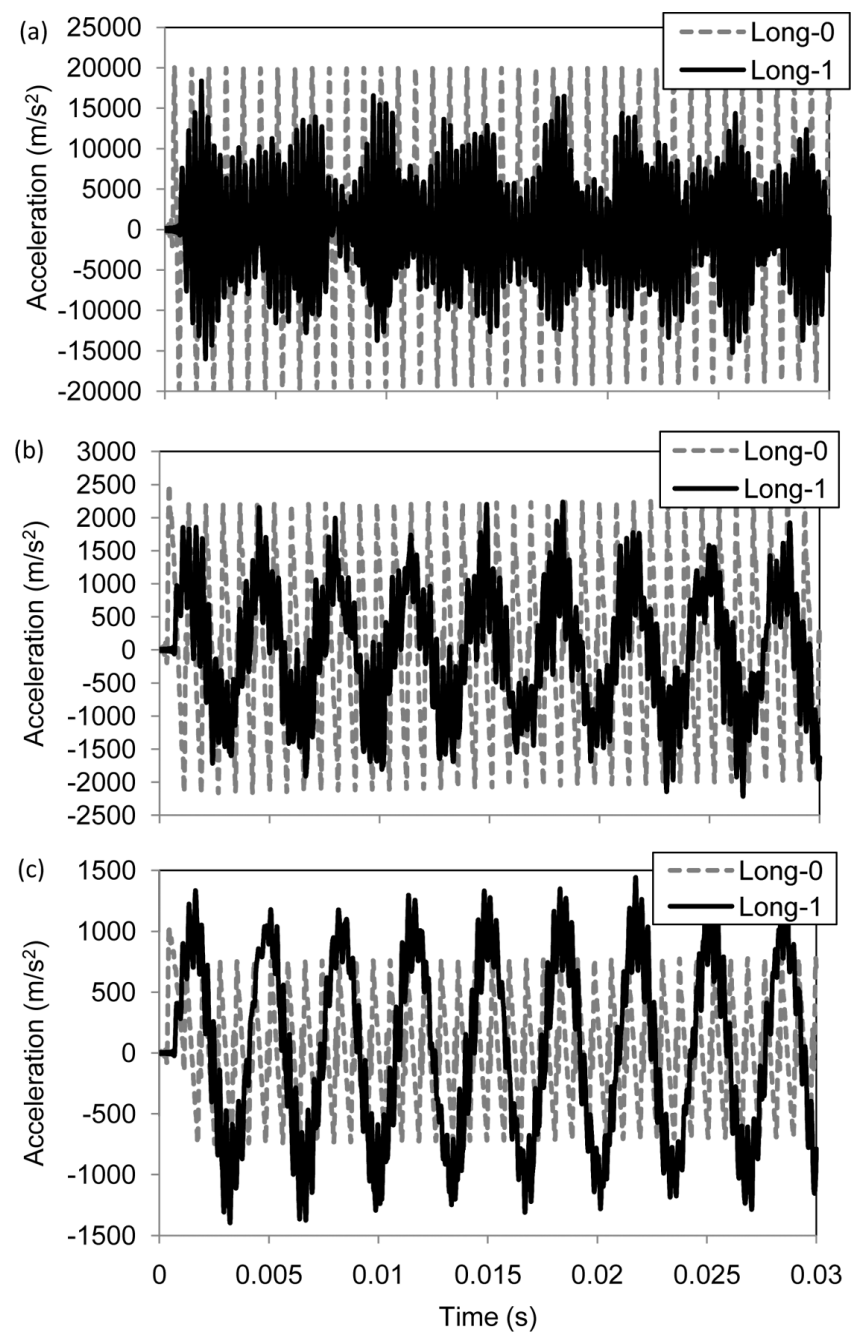

Fig. 16. Computational results of acceleration responses obtained by applying three different impact forces to long structures. Accelerations obtained using (a) Force 1; magnitude of 5,300 N with 0.0003 seconds impact duration, (b) Force 2; magnitude of 1,766 N with 0.0009 seconds impact duration and (c) Force 3; magnitude of 1,135 N with 0.0014 seconds impact duration. 10,000 Hz cutoff frequency is used for low-pass filter.

(Long-1), the acceleration was reduced compared to the structure without an interface (Long-0). This contradiction might be related to length of structures and impacts. After wave starts traveling and reaches at the other end of the structure, it does not stop there but comes back to the original point where the impact is applied. If the length of the structure is short or impact duration is long, the returning wave might be quick enough to interfere with the applied impact. In that case, the interference might cause an increase in acceleration wave. In the short structures, 'Short-0', 'Short-1' and 'Short-2' have wave propagation time of one lap (going from one end to the other end and coming back to the original point where the impact is applied) of about $6.1 \mathrm{E}-5,1.1 \mathrm{E}-4$ and $1.1 \mathrm{E}-4$ seconds, respectively, while the durations of impact are $0.2 \mathrm{E}-3$ seconds for hard and 0.6E-3 seconds for medium tip impacts. Therefore, all the combinations have interference between the applied impact and the propagating acceleration wave. In contrast to the short structures, all the combinations of the long structures and the impacts do not have any interference between the applied impact and the propagating acceleration wave. Wave propagation time of one lap is about 7.7E-4 seconds for 'Long-0' and 1.4E-3 seconds for 'Long-1', while the durations of impact using the hard and medium tips are $0.3 \mathrm{E}-3$ and $0.6 \mathrm{E}-3$ seconds, respectively.

We observed that, in the FFT responses of applied impact forces, higher the magnitude of impact force, higher 
Table 10

Excitation frequencies $(\mathrm{Hz})$ observed in FFT response of acceleration results obtained from the long structural models with three different applied impact forces. Frequencies are given in descending order of their magnitude

\begin{tabular}{|c|c|c|c|c|c|}
\hline \multicolumn{2}{|c|}{ Force 1} & \multicolumn{2}{|c|}{ Force 2} & \multicolumn{2}{|c|}{ Force 3} \\
\hline Long-0 & Long-1 & Long-0 & Long-1 & Long-0 & Long-1 \\
\hline 2,620 & 3,540 & 1,310 & 300 & 1,310 & 300 \\
\hline 1,310 & 3,800 & 2,620 & 3,540 & 6,550 & 3,540 \\
\hline 3,930 & 3,170 & 6,550 & 1,180 & & \\
\hline 6,550 & 7,060 & 7,850 & 3,170 & & \\
\hline 7,850 & & & & & \\
\hline
\end{tabular}

was the excitation frequency range. On the other hand, the structures made of all aluminum such as 'Short-0' have higher Eigen frequencies of the first excitation frequency of axial mode compared to two-material structures (Short-1 and Short-2). This was true in long structures as well. Therefore, it appears that by increasing the impact forces, one-material structures (Short-0 and Long-0) become easier to excite causing higher acceleration responses compared to two-material structures. However, we found that increasing the magnitude of impact forces did not cut out lower frequencies from the range of excitation frequencies in FFT responses. Hence, there should be an equal possibility to excite low and high excitation frequencies in structures. For instance, medium tip impact applied on the short structures had the excitation range of up to $8,000 \mathrm{~Hz}$ including the excitation frequencies observed in most of the structural configurations except 'Short-0' with $34 \mathrm{~N}-\mathrm{m}$ bolt torque. However, 'Short-0' with $34 \mathrm{~N}-\mathrm{m}$ bolt torque did not have lower acceleration compared to 'Short-0' with $21 \mathrm{~N}-\mathrm{m}$. On the other hand, 'Force 2' applied on the long structural model had the excitation frequency range of 1,600 Hz. Eigen frequencies of axial mode for 'Long-1' were 174, 296, 420, 1,160, 2,190, $3110 \mathrm{~Hz}$ and higher while the excitation frequencies observed in the FFT response were 300, 3,540,1,180 and 3,170 in the descending order of their magnitude. Therefore, excitation frequencies in the FFT responses are not necessarily within the FFT response of applied impact force. Additionally, it cannot be concluded that Eigen frequencies within a frequency range of applied impact were more easily excited than those outside the frequency range. Therefore it is hard to conclude that the acceleration differences were caused by the differences in frequency range of applied impact.

In order to explain the cause of acceleration differences, the effect of interference between an applied impact and reflected wave in acceleration responses is studied further using the Finite Element Analysis. First, simulations applying the experimentally obtained impact are conducted to verify the model. The computational results of short and long structures showed similar accelerations compared to the experiments. One noticeable difference was that the experiments show clear damping effect in acceleration response while that was not the case in the simulations. The model used in the study did not include damping as well as bolts connections. Therefore, the model does not have any material damping or physical means to reduce vibrations other than impedance mismatch. The other thing that needs to be pointed out was that the excitation frequencies in the short structures were significantly different between the simulation and experimental results, especially for 'Short-0' case. This might be contributed by two factors. First, the material properties used in the simulations are reference values obtained from literature since exact values are not available. It is possible that some of the material properties might be slightly different compared to the ones used in the experiment. Especially, Young's modulus and density affect the excitation frequency. The second cause might be from the simplification of the model. Since the structures were modeled as simple one solid structure without bolts and with common nodes at the interface, that might be contributing to the differences in excitation frequencies. As Aoki [23] reported, natural frequency is lower for the specimen with bolted joint compared to the one without bolted joint. Bolt effect is further demonstrated by the experimental results. When the torque was increased to tighten the bolts to assemble the experimental structures, peak frequencies in FFT response shifted to higher values. This phenomenon was also reported by Augustaitis et al. [24]. This reasoning was also supported by the results obtained from our long structure data. Components of the long structure were directly fastened by thread rather than using the bolts. FFT response of the computational and experimental results of long structure showed only a slight difference in excitation frequencies. Furthermore, Eigen frequencies of axial mode in the long structure obtained from the simulation matched well with the excitation frequencies obtained from FFT responses of the simulation as well as experimental accelerations, where as for short structures, they matched well with the simulation results but not with the experimental results. 
Three different impact forces are applied in the short and long structural model to verify the hypothesis (when an applied impact force interfere with the propagating wave, impedance mismatch does not help to reduce acceleration). In the experimental condition of the short structure, the applied impact and the reflected wave interfered for all three cases, 'Short-0', 'Short-1' and 'Short-2'. When the impact duration is reduced such that the applied impact and the propagating wave do not interfere, 'Short-2' has the lowest acceleration closely followed by 'Short-1'. 'Short-0' clearly shows highest acceleration among these three configurations. In contrast to the short structure, the applied impact and the propagating wave did not interfere for both 'Long-0' and 'Long-1' in the experimental conditions of long structures and the results showed clear reduction in acceleration response of 'Long-1' compared to 'Long-0'. Therefore, the impact duration is increased to have interference between the applied impact and the propagating wave in the models. Simulation results clearly showed that when the applied impact and the propagating wave interfere, 'Long-1' have higher acceleration than that of 'Long-0'.

The other noticeable point is, when only one material structure has interference between the applied impact and the propagating wave, the acceleration results of one material and two material structures show almost the same magnitude for both short and long structural cases. In other words, a structure made of one material seems to be less affected by interference between the applied impact and propagating wave. This may be explained by a number of propagating waves in a structure. Propagating wave increases its number when an interface is encountered. Therefore, possibility of interference between an applied impact and propagating wave increases. This might be one of the reasons that difference in acceleration response between 'Short-2' and 'Short-1' was very small. Additionally, when there is an interface within a structure, wave reflects back earlier at the interface than one material structure. For instance, single lap travel time of 'Short-0' is 6.1E-5 seconds while 'Short-1' and 'Short-2' are 1.1E-4 seconds. However, an interface between Aluminum and Polycarbonate for 'Short-1' reflects wave at 1.0E-5 seconds and for 'Short-2' at 5.1E-6 seconds. Therefore, not only number of propagating wave increases but also the number of interferences between applied impact and reflected wave since wave can come back quicker in 'Short-1' or 'Short-2' than 'Short-0'. It still needs further study to clarify if interferences work toward increasing or attenuating effects in acceleration responses under different configurations.

\section{Conclusions}

This report discusses the experimental and computational studies of acceleration response in the short and long cylindrical structures having impedance mismatch. The acceleration responses showed the effect of impedance mismatch based on an impact duration with respect to the length of a structure. Experimental results showed significant reduction in accelerations using two different materials if the structure was long enough that the reflective wave did not interfere with the applied impact. However, if the structure was short and the reflective wave interfered with the applied impact, the results showed increase in accelerations using two different materials. Similar trend was observed in the computational results. The short impact duration (compared to experimental force) was applied to the short cylindrical structure to avoid the interference between the reflective wave and the applied impact. The acceleration in the structure having impedance mismatch was less compared to the structure without impedance mismatch. On the other hand, the long impact duration was applied to the long cylindrical structure to see the effect of interference between the reflected wave and the applied impact. The structure without impedance mismatch showed less acceleration than that of the structure having impedance mismatch. Both experimental and computational results agreed that a material impedance mismatch helps to damp accelerations when an applied impact terminates before reflective wave interferes with the applied impact. Changes of impedance induced by layered cylinders generate both dissipation and reflection waves at the interfaces. From our results, a reflection appears to be the predominant phenomenon as explained by interference between an applied impact and reflective waves. However, more research needs to be done to provide concluding evidence.

\section{Acknowledgment}

The authors gratefully acknowledge the support of the Army Research Laboratory through the Soldier's Future Force Electronics Reliability and Survivability Technology Program, cooperative agreement number DAAD19-032-0007. 


\section{References}

[1] Z. Osiński, eds, Damping of Vibrations, A.A. Balkema, Rotterdam, 1998.

[2] J.W. Fraser and R.S. Gureghian, Controlling shock and vibration in electronic products, Mechanical Engineering 115 (1993), $82-84$.

[3] G.W. Frost and M.F. Costello, Control authority of a projectile equipped with an internal unbalanced part, Army Research Laboratory, ARL-CR-555, 2004.

[4] M.J. Wilson, R.A. Hall and M. Ilg, ONBORT (On-board Navigation of Ballistic ORDnance): Gun-launched munitions flight controller, Army Research Laboratory, ARL-TR-3210, 2004.

[5] D.S. Steinberg, Vibration Analysis for Electronic Equipment, (3rd ed.), John Wiley \& Sons, Inc., New York, 2000.

[6] A.M. Veprik and V.I. Babitsky, Vibration protection of sensitive electronic equipment from harsh harmonic vibration, Journal of Sound and Vibration 238 (2000), 19-30.

[7] G. Solaroli, Z. Gu, A. Baz and M. Ruzzene, Wave propagation in periodic stiffened shells: spectral finite element modeling and experiments, Journal of Vibration and Control 9 (2003), 1057-1081.

[8] M. Toso and A. Baz, Wave propagation in periodic shells with tapered wall thickness and changing material properties, Shock and Vibration 11 (2004), 411-432.

[9] Y. Li, K.T. Ramesh and E.S.C. Chin, Dynamic characterization of layered and graded structures under impulsive loading, International Journal of Solids and Structures 38 (2001), 6045-6061.

[10] A. Tasdemirci and I.W. Hall, The effects of plastic deformation on stress wave propagation in multi-layer materials, International Journal of Impact Engineering 34 (2007), 1797-1813.

[11] C.W. Bert, Research on dynamic behavior of composite and sandwich plates - IV, The Shock and Vibration Digest 17 (1985), 3-15.

[12] S. Abrate, Impact on laminated composites: Recent advances, Applied Mechanics Reviews 47 (1994), $517-544$.

[13] R.J. Wasley, Stress Wave Propagation in Solids, an Introduction, Marcel Dekker, Inc., New York, 1973.

[14] H.R. Harrison and T. Nettleton, Advanced Engineering Dynamics, John Wiley \& Sons, Inc., New York, 1997.

[15] D.T. Blackstock, Fundamentals of Physical Acoustics, John Wiley \& Sons, Inc., New York, 2000.

[16] M.R. Chowdhury and A. Tabiei, Development of an air gun simulation model using LS-DYNA, Army Research Laboratory, ARL-TR-3016, 2003.

[17] E.A. Szymanski, Acquiring Data for the Development of a Finite Element Model of an Airgun Launch Environment, Army Research Laboratory, ARL-MR-581, 2004.

[18] A. Bouland and M.R. Chowdhury, Analytical simulation and verification of air gun impact testing, Army Research Laboratory, ARL-TR$3559,2005$.

[19] Livermore Software Technology Corporation, LS-DYNA Keyword user's manual, Version 970, Livermore, California, 2003.

[20] J.O. Hallquist (Comp.), LS-DYNA Theory manual. Livermore, California, 2005.

[21] R.A. Ibrahim and C.L. Pettit, Uncertainties and dynamic problems of bolted joints and other fasteners, Journal of Sound and Vibration 279 (2005), 857-936.

[22] M.H. Pashaei, M.R. Davoodi and H. Nooshin, Effects of tightness of bolts on the damping of a MERO-type double layer gird, International Journal of Space Structures 21 (2006), 103-110.

[23] S. Aoki, Dynamic characteristics of structure with bolted joint considering some factors, ASME Pressure Vessels Piping Div Publ PVP (2004 ASME/JSME Pressure Vessels and Piping Conference. San Diego, CA) 486 (2004), 143-149.

[24] V.K. Augustaitis, V. Bučinskas and V. Pauža, Application of Frequency Method for Defining Threaded Joint Tightening Degree, Solid State Phenomena 113 (2006), 277-280.

[25] M.S. Berman, The effect of transient loading discretization on a rotating disk using Lawrence Livermore National Laboratories' Dyna 3-D. Army Research Laboratory, ARL-MR-557, 2003. 

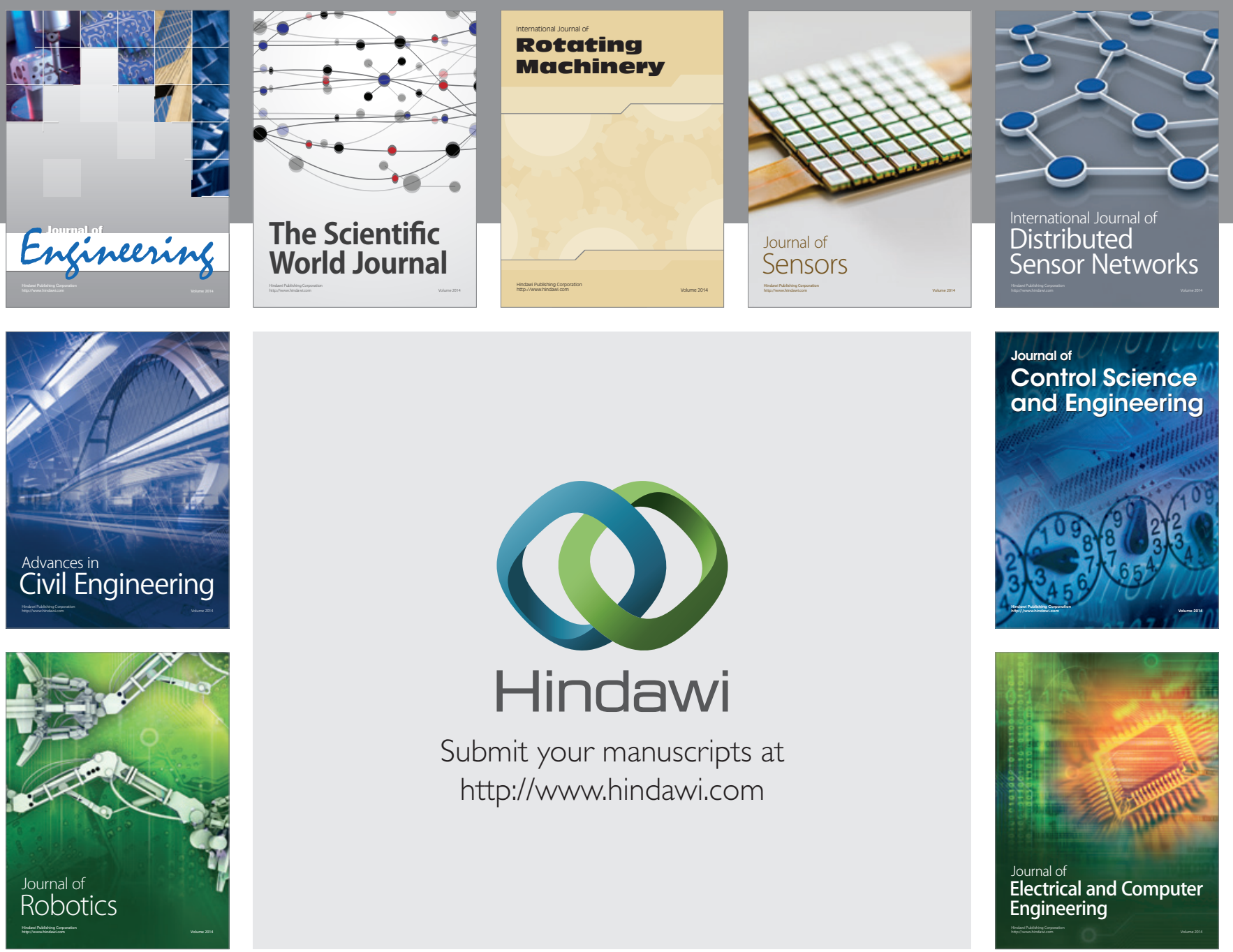

Submit your manuscripts at

http://www.hindawi.com
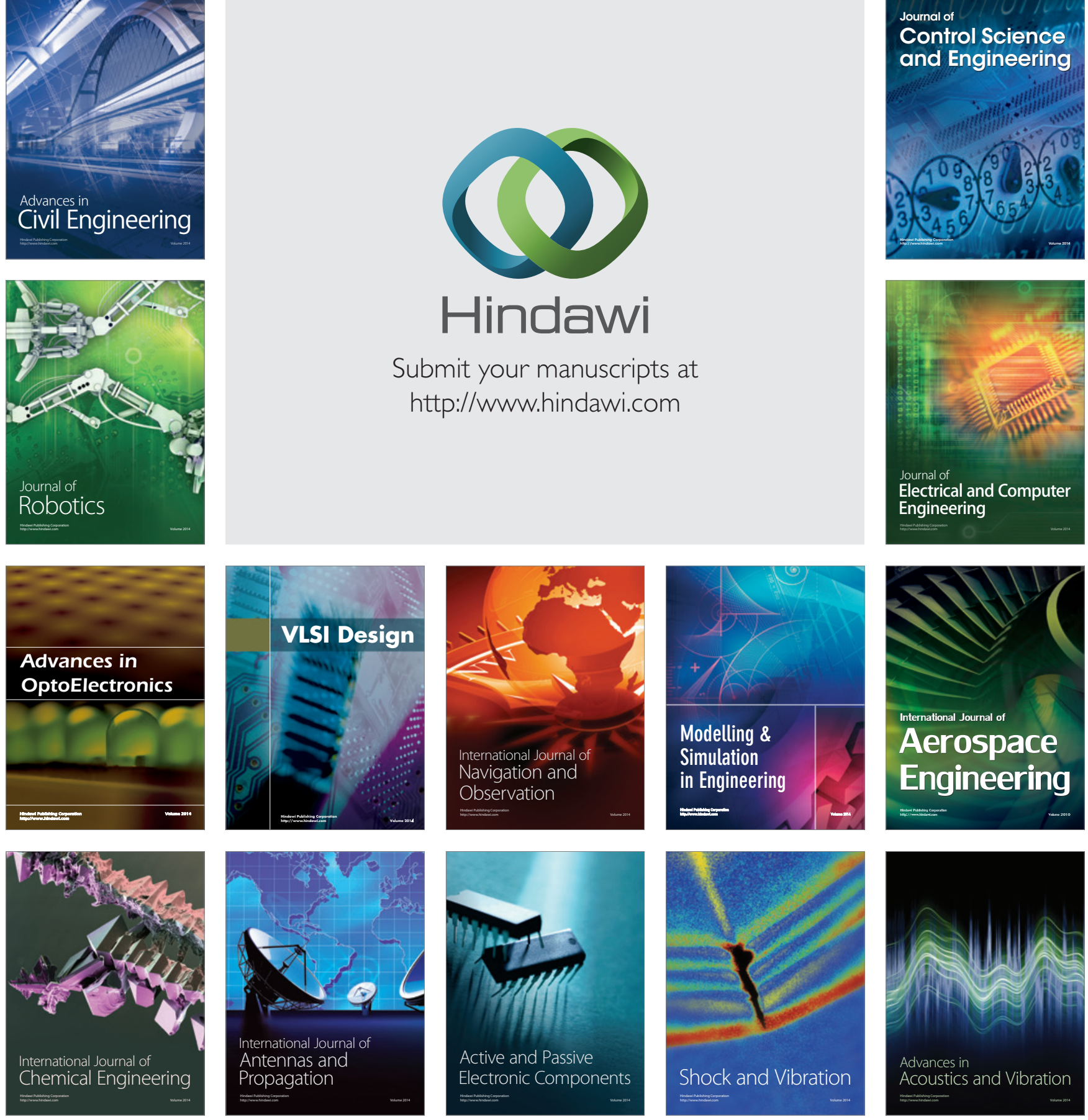